\title{
Discrete Polar Complex Exponential Transform for Image Rotation Duplication Detection
}

\author{
${ }^{1}$ Junliu Zhong and ${ }^{2}$ Yanfen Gan \\ ${ }^{1}$ School of information engineering \\ Guangdong mechanical \& electrical college \\ Guangzhou, 510550 p.r china \\ ${ }^{2}$ Department of information science and technology \\ guangdong university of foreign studies south china business college \\ guangzhou, 510540 p.r china \\ junliuzhong@foxmail.com,fannygyf@foxmail.com
}

\begin{abstract}
With the aid of powerful photo editing software, region duplication is a easy way to create digital forgery images. Many prior approaches to detect and locate similar or identical regions are introduced in various application fields successfully. But in most case, these existing region duplication detection approaches can only detect some simple forensics which cloned region is moved to a different position in the same image, and are not effective when the duplicated regions have been subjected to geometrical distortions and added noise. In this paper, we describe a new region duplication detection approach that is robust to rotation of the duplicated regions. We propose an algorithm by constructing rotation moment invariants and extracting invariance features of images for Two-Dimensional (2-D) image forgery fields based on Discrete Polar Complex Exponential Transform (DPCET) which is one of three types of Discrete Polar Harmonic Transforms (DPHTs). This Discrete Polar Complex Exponential Transform approach is improved from traditional Polar Complex Exponential Transform (PCET) and this transform also encompasses the features of orthogonality and rotation invariance. We introduce a new $9 \times 9$ circle instead of a unit circle in DPCET to calculate features of image more precision. And various experiments were performed to prove that effectiveness of our approach in detecting duplicated forgeries, with translation, rotation, noise addition. And the experiment results also show that our approach is more robust to geometric distortions of the duplicated regions than other compared approaches.
\end{abstract}

Keywords: digital forgery images, duplication detection, rotation invariance, Discrete Polar Complex Exponential Transform, Discrete Polar Harmonic Transforms, 9×9 circle

\section{Introduction}

Over the past decade, information forensics and security become popular and attract much more people's attention. Image forensics is a key problem of information forensics and security field in today's society. Hence, image forgery detection is an important area of research in the field of social livelihood, scientific discovery, court evidence, financial document, news reports et al. With the development of the powerful photo processing software, some malicious users can perform forgery images to achieve their desired purposes. A common manipulation in tampering with digital images is known as region duplication, where a continuous portion of pixels are copied and pasted to a different location in the same image in order to conceal an important object in the scene [1]. Forgery meeting between President Clinton and Saddam Hussein is shown in Figure 1. 
We focus on image forgery detection and aim at establishing correspondence between the same or similar duplicated parts which appear in one image or other different images.

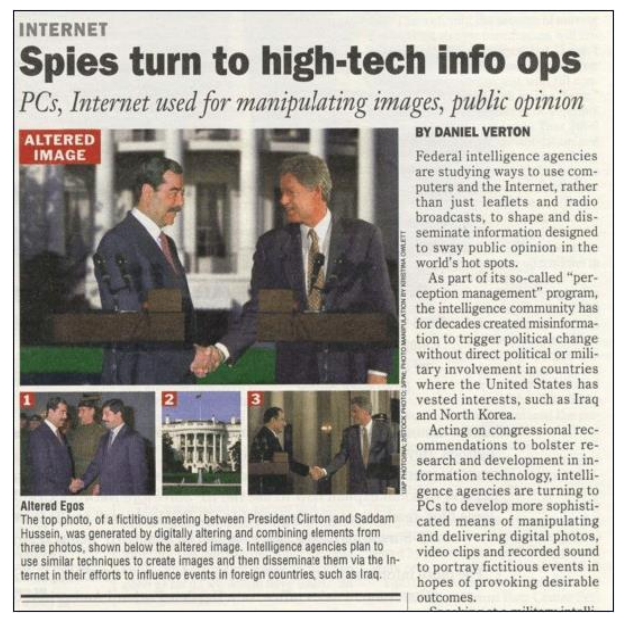

\section{Figure 1. Forgery Duplicated Image of Meeting between President Clinton and Saddam Hussein}

Some general approaches are proposed to detect and locate duplicated forgery images. Fridrich [2] proposed approach base on Discrete Cosine Transform (DCT) coefficients which is one of the most widely-used techniques for duplicated forgery detection. But this approach has a low efficiency. In order to increase efficiency, Popescu et al. [3] proposed an approach based on the principal component analysis (PCA). PCA approach can reduce dimensionality of the matrix and get the aim to reduce computational complexity and cost. Cao et al. [4] and Huang et al. [5] also proposed an improved DCT mean coefficient approach and aimed to reduce the dimension of the feature vectors used by the Fridrich's algorithm. Zhang et al. [6] proposed an approach based on the pixel-matching for detecting and locating Copy-Move regions within an image. Chakraborty et al. [7] proposed an approach to joint histogram and mutual information for detecting illumination change in duplicated region. Kashyap et al. [8] proposed an approach based on wavelet decomposition.

The above detection approaches can detect forgeries in images well in the absence of post-processing operations. But this is not always the case. These general detection approaches typically do not provide valid evidence of region duplication or the location of the duplicated regions when the duplicated regions are suffered from geometric distortion. Considering image geometric distortion with translation, rotation and mirror is a key factor of image quality. Due to geometric invariant moments are highly concentrated on geometric transformation of image features, therefore, in the field of image duplication detection, geometric transformation (rotation, translation and scaling) invariance analysis is an important research content. In recently years, research on geometric distortion of forgery duplication image has been obtained fruitful achievements in the study of geometric moment invariants. Various kinds of moment invariants are proposed in the past decades. HU [9] moments are first proposed and $\mathrm{Hu}$ moments are widely-used techniques for duplicated forgery detection. Teh [10] ever proposed and evaluated a number of moments and addresses some fundamental questions. Latter various types of moment invariants are appeared to detect duplicated region of images. Popular and representative moment invariants approached are divided into several groups.

Scale Invariant Feature Transform (SIFT)

Amerini [11] proposed Scaling-Invariant-Features-Transform (SIFT) to reliably detect digital forgery images even if it is suffered from a large scaling transform. Pan et al. [1] proposed an approach by estimating the transform between matched SIFT feature key 
points, which are insensitive to geometrical and illumination distortions, and then finds all pixels within the duplicated regions after discounting the estimated transforms. Sedaghat et al. [12] proposed a fully automated matching algorithm, called uniform robust SIFT, namely UR-SIFT. Chihaoui et al. [13] proposed to use the SIFT approach and match identical features by using the Singular Value Decomposition (SVD) approach to detect duplicated local characteristics or interest points of the images.

\section{2) Zernike Moments (ZMs)}

Ryu et al. [14] proposed Zernike moments which used as robust invariant features and then are able to detect accurately duplicated regions. When only rotation of the copied region takes place, the approach is superiority. Revaud et al. [15] proposed an accurate rotation-invariant similarity measure while retrieving the optimal in-plane rotation angle. Chen et al. [16] proposed an approach to derive a set of combined geometric-blur invariants based upon orthogonal Zernike moments (OZMs). LI et al. [17] propose combining both the magnitude and phase coefficients to form a new shape descriptor, referred to as invariant ZM descriptor (IZMD).

\section{3) Fourier Mellin Moments (FMMs)}

Bayram et al. [18] proposed to extract image characteristics from blocks of image by applying the Fourier Mellin Transform (FMT). Sheng et al. [19] proposed Orthogonal Fourier-Mellin moments (OFMMs) to describe rotation invariant patterns. Singh [20] propose a new approach to construct rotation invariant which combines the merits of the Fourier-Mellin-Transform (FMT) and Modified Phase-Only Correlation (MPOC) techniques. The experiment results show that this approach has a high resolution in partial palm print images detection which is rotated and added Gaussian noise.

\section{4) Polar Harmonic Transforms (PHTs)}

Yap [21] introduced a set of 2D of transforms, namely Polar Harmonic Transforms (PHTs) in 2010. PHTs are a set of 2D transforms defined over the unit circle in the polar coordinate system which can be used to generate rotation-invariant features. Li et al. [22] used PHTs to extract the features of the circular blocks, which are then used to perform block matching and locate duplicated region of the forgery image. PHTs [21-23] encompass the orthogonality and invariance which is superiority to ZMs, PZMs and SIFT. Compared ZMs, PZMs, SIFT and OFMM, computation of PHTs kernel is simple and stable [21]. Hence PHTs have provided a powerful tool for image detection.

In this paper, we do research on image forgery field, mainly focus on the duplicated region detection. The essential challenge in duplicated region detection field is how to detect, recognize and locate one object in an image location. We propose a new Discrete Polar Complex Exponential Transform which is belong to Discrete Polar Harmonic Transforms approach to construct translation, rotation moment invariants and extract translation, rotation invariance to detect duplicated region, even if these region are subjected to geometric distortion, such as translation, a large degree rotation tampered, and additive noise. We introduce a new $9 \times 9$ circle instead of a unit circle in DPCET to calculate features of image more precision.

The rest of the paper is organized as follow: in Section 2, we review the Two-Dimension Polar Harmonic Transforms. In Section 3, we propose our approach and define Discrete Polar Harmonic Transforms (DPHTs), mainly DPCET. Detail to discuss DPCET to construct rotation moment invariants and extract region invariance features. In Section 4 we describe a few experiments which results we have performed to evaluate the performance of the proposed approach. We further demonstrate its practical performance by introducing Image False Detecting Rate (FDR) and comparing with other approached in detecting precision rate. Finally, the conclusions are given in Section 5. 


\section{The Review of the 2-D Polar Harmonic Transforms}

In this section, we have grouped three types' transforms which is Polar Complex Exponential Transform (PCET), Polar Cosine Transform (PCT), and Polar Sine Transform (PST) under the name Polar Harmonic Transforms as the kernels of these transforms. These three type transforms are harmonic basic waves of PHTs.

\subsection{Definition of Polar Complex Exponential Transform (PCET)}

The general 2-D moment definition using a moment weighting kernel, namely basis function $H_{n l}(x, y)$, and an image intensity function $f(x, y)$ is given as

$$
M_{n l}=\frac{1}{\pi} \int_{0}^{2 \pi} \int_{0}^{1} H_{n l}^{*}(x, y) f(x, y) d x d y
$$

Given a 2D image function $f(r, \theta)$, it can be transformed $f(x, y)$ to it from Cartesian coordinate to Polar coordinate. The transforms in this set of function $f(r, \theta)$ is defined as $[21,22]$

$$
M_{n l}=\frac{1}{\pi} \int_{0}^{2 \pi} \int_{0}^{1} H_{n l}^{*}(r, \theta) f(r, \theta) r d r d \theta
$$

Where $M_{n l}$ is Polar Complex Exponential Transform (PCET) of $f(r, \theta)$, with order n and repetition 1. where $|n|, l=0,1 \ldots, \infty H_{n l}(r, \theta)$ is a basis function of PCET, $H_{n l}^{*}(r, \theta)$ indicates the complex conjugate of the $H_{n l}(r, \theta)$. For PCET, $H_{n l}^{*}(r, \theta)$ is consisted of radial and circular components and can be defined as

$$
H_{n l}^{*}(r, \theta)=R_{n}^{*}(r) e^{-i l \theta}=e^{-i 2 \pi n r^{2}} e^{-i l \theta}
$$

Where $R_{n}(r)$ is the radial kernel, $i$ is a imaginary and $i=\sqrt{-1}, r$ indicates radius defined on unit circle and $\theta$ indicates rotation degree which are defined as

$$
\begin{aligned}
& r=\sqrt{x^{2}+y^{2}}, \\
& \theta=\tan ^{-1}\left(\frac{y}{x}\right)
\end{aligned}
$$

$R_{n}(r)$ is satisfy orthogonal condition, and defined as [21]

$$
\begin{aligned}
& \int_{0}^{1} R_{n}(r) R_{n}^{*}(r) r d r=\frac{1}{2} \delta_{n n}^{\prime} \\
& \int_{0}^{2 \pi} \int_{0}^{1} H_{n l}(r, \theta) H_{n l}^{*}(r, \theta) r d r d \theta=\pi \delta_{n n}^{\prime} \delta_{l l}^{\prime}
\end{aligned}
$$

\subsection{Definition of Polar Cosine Transform (PCT) and Polar Sine Transform (PST)}

Similar to the form of PCET, Polar Cosine Transform (PCT) is defined as [21]

$$
M_{n l}^{C}=\Omega_{n} \int_{0}^{2 \pi} \int_{0}^{1}\left[H_{n l}^{C}(r, \theta)\right]^{*} f(r, \theta) r d r d \theta
$$

Polar Sine Transform (PST) is defined as

$$
M_{n l}^{C}=\Omega_{n} \int_{0}^{2 \pi} \int_{0}^{1}\left[H_{n l}^{S}(r, \theta)\right]^{*} f(r, \theta) r d r d \theta
$$

Where the basis function of PST and PCT are defined as

$$
\left\{\begin{array}{l}
H_{n l}^{C}=\cos \left(n \pi r^{2}\right) e^{i l \theta} \\
H_{n l}^{S}=\sin \left(n \pi r^{2}\right) e^{i l \theta}
\end{array}\right.
$$


Where $\Omega_{n}=\left\{\begin{array}{ll}\frac{1}{\pi}, & \text { if } n=0 \\ \frac{2}{\pi}, & \text { if } n \neq 0\end{array} \quad,|n|, l=0,1 \ldots, \infty\right.$

\section{Proposed Approach based on Discrete Polar Complex Exponential Transform}

In this section, we have mainly introduced and developed Discrete Polar Complex Exponential Transform (DPCET). And Discrete Polar Cosine Transform (DPCT) and Discrete Polar Sine Transform (DPST) is similar to be constructed. The proposed approach constructs rotation moment invariants based on the Polar Complex Exponential Transform. This approach by extracting translation, rotation features can allow it to detect and locate duplicated region which are undergone post processing, such as rotation, noise addition tampered.

\subsection{Constructing Rotation Moment Invariant}

From (2), we supposed $\theta=\phi+\beta$ and get derivation $d \theta=d \phi$. We also supposed $g(r, \theta)=f(r, \phi)$, Where $g(r, \theta)$ is $f(r, \phi)$ rotated a $\beta$ degree. Hence, (2) can be rewritten as

$$
\begin{aligned}
& M_{n l}^{R}=\frac{1}{\pi} \int_{0}^{2 \pi} \int_{0}^{1} H_{n l}^{*}(r, \theta) g(r, \theta) r d r d \theta \\
& =\frac{1}{\pi} \int_{0}^{2 \pi} \int_{0}^{1} \exp \left[-i\left(2 \pi n r^{2}+l \theta\right)\right] g(r, \theta) r d r d \theta \\
& =\frac{1}{\pi} \int_{0}^{2 \pi} \int_{0}^{1} \exp \left(-i 2 \pi n r^{2}\right) \exp [-i l(\phi+\beta)] f(r, \phi) r d r d \phi \\
& =\frac{1}{\pi} \exp (-i l \beta) \int_{0}^{2 \pi} \int_{0}^{1} \exp \left[-i\left(2 \pi n r^{2}+l \phi\right)\right] f(r, \phi) r d r d \phi
\end{aligned}
$$

(10) is also rewritten as

$$
M_{n l}^{R}=M_{n l} e^{-i l \theta}
$$

From (10), (11) we can construct a rotation moment invariant as $\left|M_{n l}^{R}\right|=\left|M_{n l} e^{-i l \theta}\right|=\left|M_{n l}\right|$

\subsection{Definition of Discrete Polar Complex Exponential Transform (DPCET) and Rotation Invariance Extraction}

Given a 2D signal function ${ }^{-}(x, y)$, the Polar Complex Exponential Transform is defined on a unit circle in Cartesian coordinate as [21].

$$
M_{n l}=\frac{1}{\pi} \iint_{x^{2}+y^{2} \leq 1}\left[\bar{H}_{n l}(x, y)\right]^{*} \bar{f}(x, y) d x d y
$$

Where $\bar{f}(x, y)=\bar{f}(r \cos \theta, r \sin \theta)=f(r, \theta)$

and $\bar{H}_{n l}(x, y)=\bar{H}_{n l}(r \cos \theta, r \sin \theta)=H_{n l}(r, \theta)$

The Polar Complex Exponential Transform function defined on a unit circle is shown in Figure 2. 


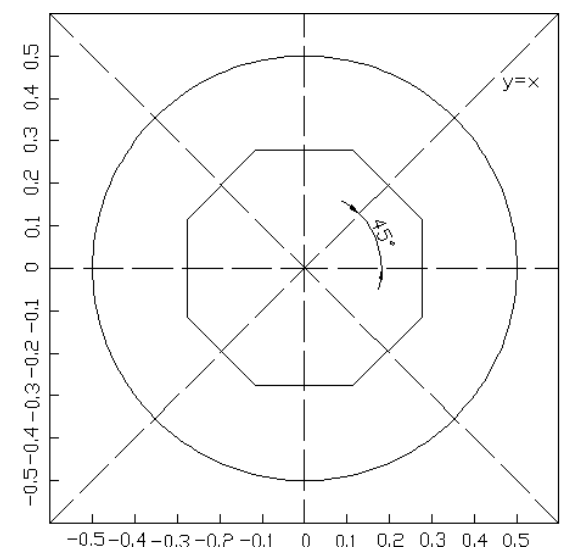

\section{Figure 2. The Polar Complex Exponential Transform Function Defined into a Unit Circle}

The above discussion has been only limited to apply in continuous image domain. In practice, this is not always a case. Images which we obtain are defined on a discrete domain. Given an image with size of $P \times Q$, (14) shows the image is necessary for proper normalization. Hence, its pixels are mapped on discrete unit circle domain. Supposed $p=0,1, \ldots P$ and $q=0,1, \ldots, Q$.

$x_{p}=\frac{2 p-P}{P}, y_{q}=\frac{2 q-Q}{Q}$

Hence, discrete of (10) can be defined as

$$
M_{n l}=\frac{1}{\pi} \sum_{p=0}^{P} \sum_{q=0}^{Q}\left[\bar{H}_{n l}\left(x_{p}, y_{q}\right)\right]^{*} \bar{f}\left(x_{p}, y_{q}\right) \Delta x \Delta y
$$

Supposed $_{\Delta x}=\frac{2}{P}, \Delta y=\frac{2}{Q},(13)$ and (15) can also be written as

$$
\begin{aligned}
& M_{n l}=\frac{4}{\pi P Q} \sum_{p=0}^{P} \sum_{q=0}^{Q}\left[\bar{H}_{n l}\left(x_{p}, y_{q}\right)\right]^{*} \bar{f}\left(x_{p}, y_{q}\right) \\
& =\frac{4}{\pi P Q} \sum_{p=0}^{P} \sum_{q=0}^{Q} \exp \left\{-i\left[2 n \pi\left(x_{p}^{2}+y_{q}^{2}\right)^{2}+l \tan ^{-1}\left(y_{q} / x_{p}\right)\right]\right\} \bar{f}\left(x_{p}, y_{q}\right)
\end{aligned}
$$

In our detection technique, we proposed a detected circular template which size is $9 \times 9$ to detect the image. Figure 3 is shown as

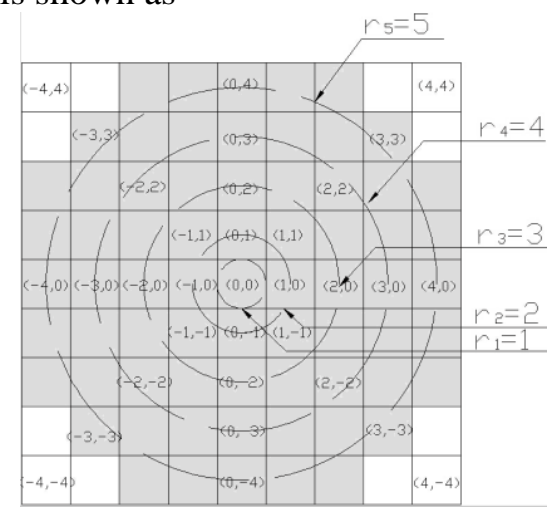

Figure 3. $9 \times 9$ Circular Template

The template is a circular block which is consisted of gray grid pixels. From Figure 3, (16) can be rewritten as

$$
M_{k l}=\frac{1}{\pi} \sum_{p=-4}^{4} \sum_{q=-4}^{4}\left[\bar{H}_{n l}\left(x_{p}, y_{q}\right)\right]^{*} \bar{f}\left(x_{p}, y_{q}\right) \Delta x \Delta y
$$




$$
\begin{aligned}
& M_{n l}=\frac{4}{\pi 81} \sum_{p=0}^{P} \sum_{q=0}^{Q}\left[\bar{H}_{n l}\left(x_{p}, y_{q}\right)\right]^{*} \bar{f}\left(x_{p}, y_{q}\right) \\
& =\frac{4}{\pi 81} \sum_{p=-4}^{4} \sum_{q=-4}^{4} \exp \left\{-i\left[2 \pi k\left(x_{p}^{2}+y_{q}^{2}\right)^{2}+l \tan ^{-1}\left(y_{q} / x_{p}\right)\right]\right\} \bar{f}\left(x_{p}, y_{q}\right)
\end{aligned}
$$

In order to calculate the $9 \times 9$ template DPCET more accuracy, we divide template into eight directions and calculate the sum of the direction each time in template which is shown in Figure 2 and Figure 3. The eighth direction is separated by $45^{\circ}$. We can rewrite (19) by using (13) and the approach in Figure 2 and Figure 3. Hence, we can define DPCET as

$$
\begin{aligned}
& \left|M_{D, n l}\right|=\left|\sum_{d=0}^{7} M_{d, n l}\right| \\
& =\left|\frac{4}{\pi 81} \sum_{d=0}^{7} \sum_{p=0}^{P} \sum_{q=0}^{Q}\left[\bar{H}_{n l}\left(x_{p}, y_{q}\right)\right]^{*} \bar{f}\left(x_{p}, y_{q}\right)\right| \\
& =\left|\frac{4}{\pi 81} \sum_{d=0}^{7} \sum_{p=-4}^{4} \sum_{q=-4}^{4} \exp \left\{-i\left[2 \pi k\left(x_{p}^{2}+y_{q}^{2}\right)^{2}+l \tan ^{-1}\left(y_{q} / x_{p}\right)\right]\right\} \bar{f}\left(x_{p}, y_{q}\right)\right|
\end{aligned}
$$

Original coordinate $(0,0)$ is a center pixel in a circular template. By (12) and (18) to calculate the RPCET of the template, we can get this center pixel the rotation invariance. And then we slide one pixel each time using other $9 \times 9$ template to detect the whole image. The $9 \times 9$ template which slide in image is shown in Figure 4. The above form of invariance can also be similarly applied to DPCT and DPST.

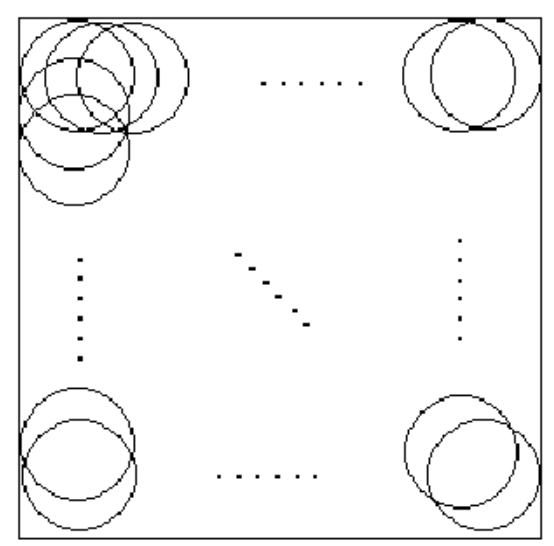

Figure 4. The $9 \times 9$ Template Slide in Image

\section{Experimental Results}

With the aid of sophisticated photo-editing software, such as Photoshop, forgery images can be made with convincing visual appearance using duplicated and distorted regions [1]. An evaluation of the detection approaches performance in image forensics field is still a difficult task. The detection of geometric distortion, especial translated, rotated duplicated forgery is still considered a challenging problem. In this section, paper results are got from an extensive series of detection and localization experiments and supported by these experimental data. We analyze, discuss the paper results in the presence of duplicated forgery images which have translated, rotated transform and added noise, and finally prove our approach the feasibility, superiority. This section is organized like this: First, we test the robustness and performance of our approach with a variety of 100 nature images which duplicated region of size of $32 \times 32$ pixels, size of $64 \times 64$ pixels and size of $128 \times 128$ pixels. We will show the detection precision rates on various 
tampered experiments which mainly are duplicated region rotated from $0^{\circ}$ to $180^{\circ}$ and added different Gaussian noise. The Fig.5 12 are performed to gauge the performance of DPCET. And then we input 100 nature images which are not tampered and use DPCET approach to detect these not being tampered images and obtain false detecting rate of these images. In the last experiment, we compare our performances with Ryu's [14] and Singh's [20]. The experimental results are shown that our approach is relatively more accuracy than compared approaches. In our experiments, MATLAB 7.0 Math Software is applied to analysis the approach in our paper and other relevant approaches.

\subsection{Detection Precision of DPCET}

We download a variety of 100 nature images from the Internet for our experiments. We take these 100 test images with a set of forgery ways by Photoshop. We tempered the image region with the ways of translation, clockwise rotation, and additive Gaussian noise. To further evaluate the performance of our approach in rotation duplicated image, we duplicate region and rotate it from $0^{\circ}$ to $180^{\circ}$. Then we get these duplicated rotation sample and test them which degrees are separated by $5^{\circ}$. Further more, the duplicated region is subdivided into three type sizes which is size of $32 \times 32$, size of $64 \times 64$ and size of $128 \times 128$. The above tampered image samples are tested with the proposed approach in this paper, and the tested samples and results are showed in Figure 5 Figure 12:

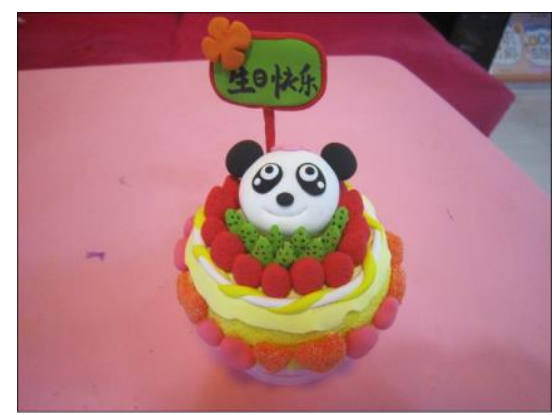

Figure 5. Origin Image of Panda-cake

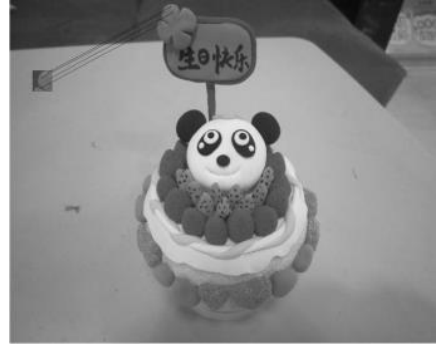

(a)

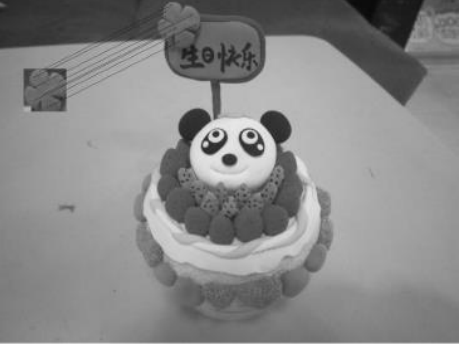

(b)

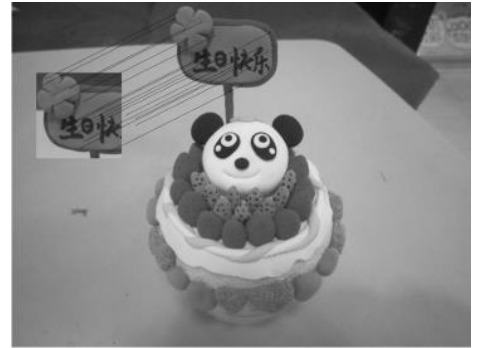

(c)

Figure 6. Detection for translation tampered $\left(0^{\circ}\right.$ clockwise rotation) and some matched pixels are connected them with lines. (a) Duplicated region size of $32 \times 32$ pixels; (b) Duplicated region size of $64 \times 64$ pixels; (c) Duplicated region size of $128 \times 128$ pixels. 


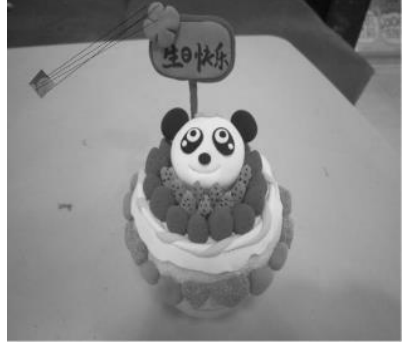

(a)

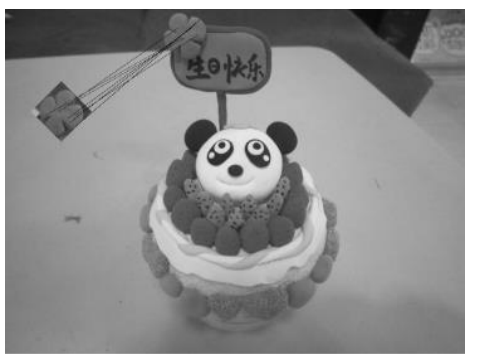

(b)

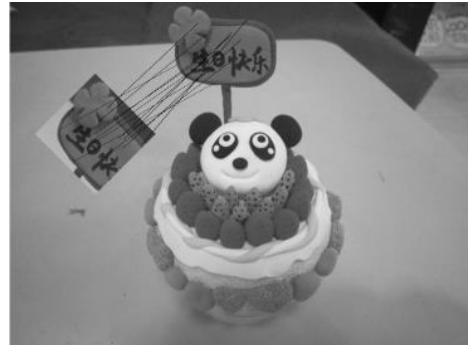

(c)

Figure 7. Detection for $45^{\circ}$ clockwise rotation tampered and some matched pixels are connected them with lines. (a) Duplicated region size of $32 \times 32$ pixels; (b) Duplicated region size of $64 \times 64$ pixels; (c) Duplicated region size of $128 \times 128$ pixels.

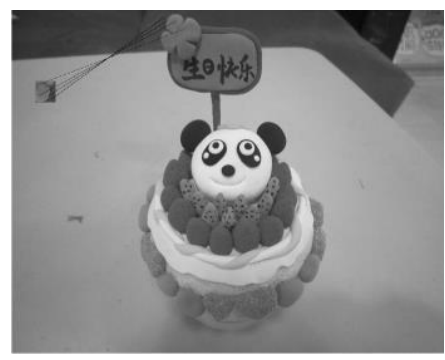

(a)

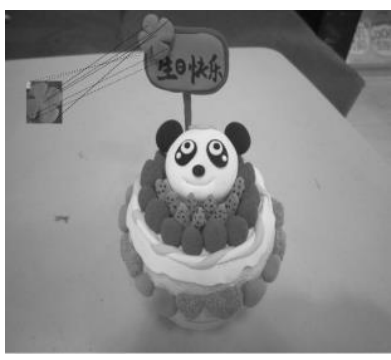

(b)

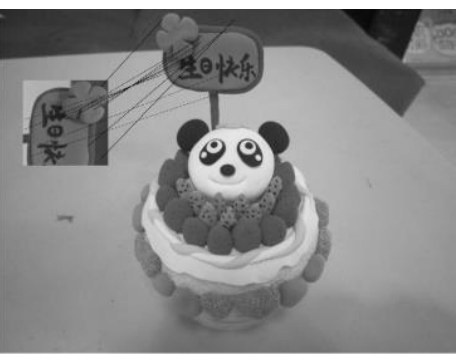

(c)

Figure 8. Detection for $90^{\circ}$ clockwise rotation tampered and some matched pixels are connected them with lines. (a) Duplicated region size of $32 \times 32$ pixels; (b) Duplicated region size of $64 \times 64$ pixels; (c) Duplicated region size of $128 \times 128$ pixels.

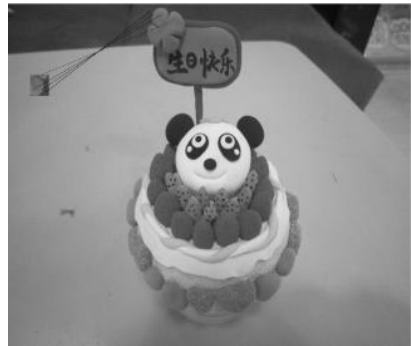

(a)

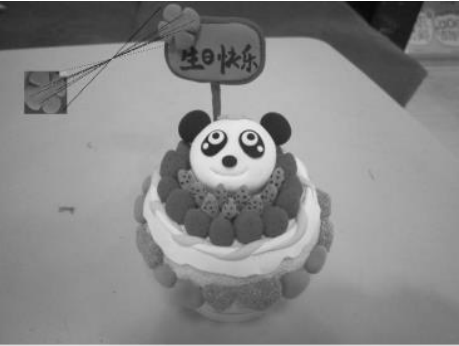

(b)

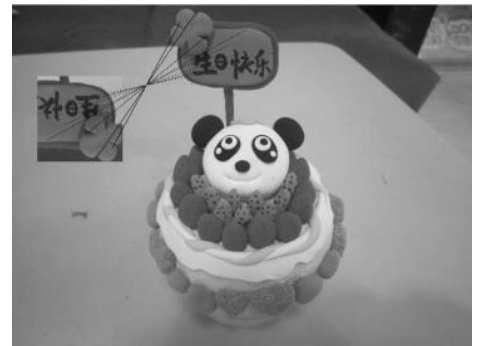

(c)

Figure 9. Detection for $180^{\circ}$ clockwise rotation tampered and some matched pixels are connected them with lines. (a) Duplicated region size of $32 \times 32$ pixels; (b) Duplicated region size of $64 \times 64$ pixels; (c) Duplicated region size of $128 \times 128$ pixels. 


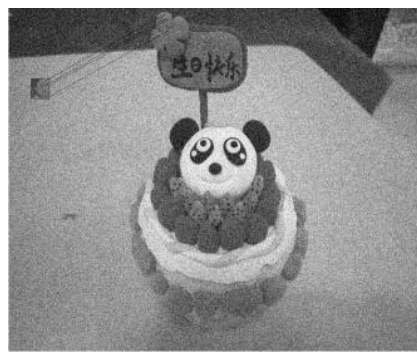

(a)

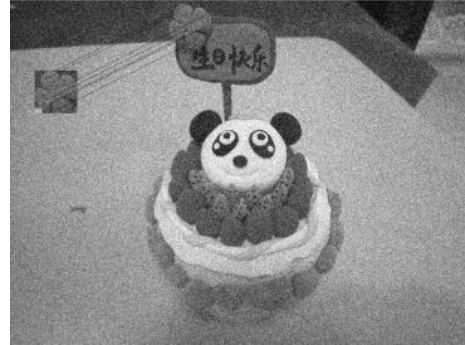

(b)

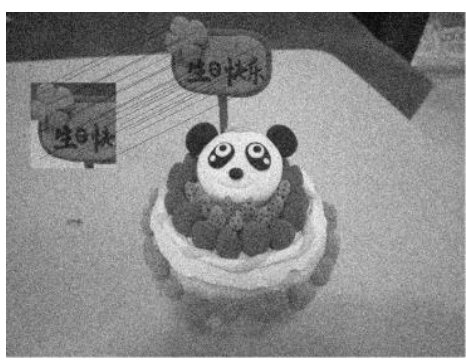

(c)

Figure 10. Detection for translation, additive Gaussian noise $\left(\mu=0.01, \delta^{2}=0.01\right)$ tampered and some matched pixels are connected them with lines. (a) Duplicated region size of $32 \times 32$ pixels; (b) Duplicated region size of $64 \times 64$ pixels; (c) Duplicated region size of $128 \times 128$ pixels.

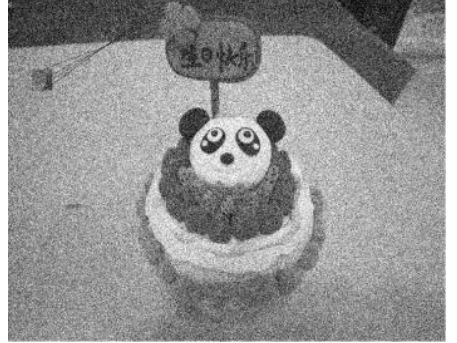

(a)

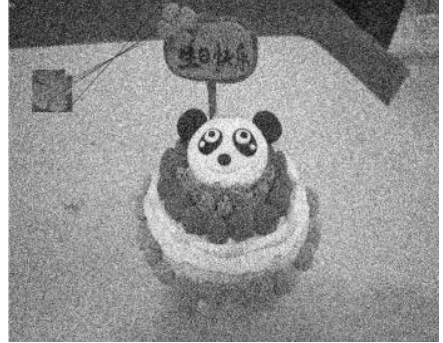

(b)

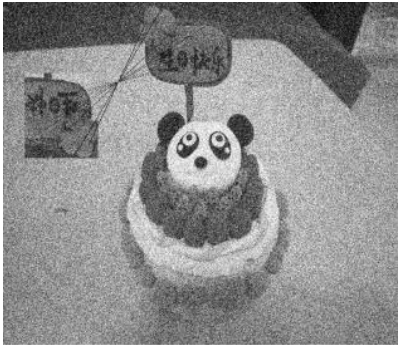

(c)

Figure 11. Detection for $180^{\circ} \mathrm{clockwise}$ rotation, additive Gaussian noise $\left(\mu=0.05, \delta^{2}=0.05\right)$ tampered and some matched pixels are connected them with lines. (a) Duplicated region size of $32 \times 32$ pixels; (b) Duplicated region size of $64 \times 64$ pixels; (c) Duplicated region size of $128 \times 128$ pixels.

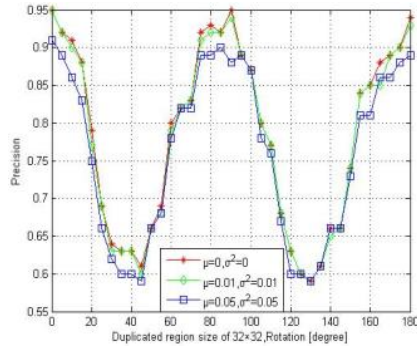

(a)

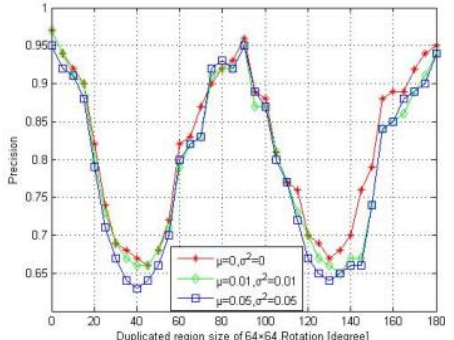

(b)

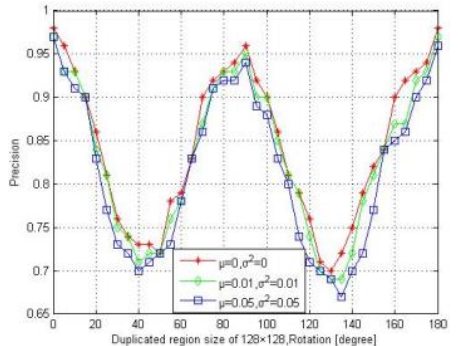

(c)

Figure 12. Detection precision rate of duplicated and tampered region with additive Gaussian noise, and rotated from $0^{\circ} \sim 180^{\circ}$ degrees (separated by $5^{\circ}$ ). (a) Duplicated region size of $32 \times 32$ pixels; (b) Duplicated region size of $64 \times 64$ pixels; (c) Duplicated region size of $128 \times 128$ pixels.

From above experimental results, our DPCET approach in detecting duplicated images which are suffered from translation and rotation will still keep certain precision rates, especially, when the rotated degree is close to $n \pi / 2$. But when the rotated degree is close to $(2 n+1) \pi / 4$, the relative detection precision rate is dramatically declined. And Additive Gaussian noise is not apparently acting on our image detection. This is because the duplicated region which is rotated close to $(2 n+1) \pi / 4$ led to the change the relatively position of neighboring pixels which will be filled a certain pixel values. And the duplicated region which is rotated close to $n \pi / 2$ is detected more easily and 
precision. When the duplicated region sizes is lager, such as $128 \times 128$, is located, detected more easily and has higher precision compared with other smaller sizes.

\subsection{Image False Detecting Rate of DPHTs in Our Approach}

From section 4.1, we can prove that our approach precision is well. But this result is established on a supposed fact that an image is tampered and exists duplicated region. In practice, this is not real case. It is usually not prior to know whether the tested image is tampered. For an nature or not being tampered image, a true case is our method does not detect any continuous duplicated region. In paper [1], region based performance measures such as pixel detection accuracy (PDA), pixel false positive (PFP) rates are useful. In this paper, in order to further demonstrate the benefit of DPHTs approach, we present image False Detecting Rate (FDR). We also input 100 nature images which are not tampered. We use DPHTs to detect these not being tampered images and obtain the image false detecting rate (FDR). The image false detecting rate is shown in Table 1.

Table 1. Average Image False Detecting Rate (Percent) for our Approach on 100 Not Being Duplicated Images under Certain Angles and Additive Gaussian noise

\begin{tabular}{|c|c|c|c|}
\hline Size & $\mathbf{3 2} \times \mathbf{3 2}$ & $\mathbf{6 4} \times \mathbf{6 4}$ & $\mathbf{1 2 8} \times \mathbf{1 2 8}$ \\
\hline Not being tampered & $2 \%$ & $0 \%$ & $0 \%$ \\
\hline Additive noise $\mu=0.01, \delta^{2}=0.01$ & $3 \%$ & $0 \%$ & $0 \%$ \\
\hline Additive noise $\mu=0.05, \delta^{2}=0.05$ & $2 \%$ & $1 \%$ & $0 \%$ \\
\hline Additive noise $\mu=0.1, \delta^{2}=0.1$ & $3 \%$ & $1 \%$ & $0 \%$ \\
\hline
\end{tabular}

Table 1 shows the image false detecting rate of our approach which is quiet well. And it refers to the size of the duplicated region which we supposed is not smaller than $\mathrm{M} \times \mathrm{N}$ $(M \geq 32, N \geq 32$ ) pixels. Judged threshold of matching pixels is not lower than $70 \% \times \mathrm{M} \times \mathrm{N}$. Other wise, it is not a true forgery image. Figure 13 shows the matching pixels which connected them with lines in an image.

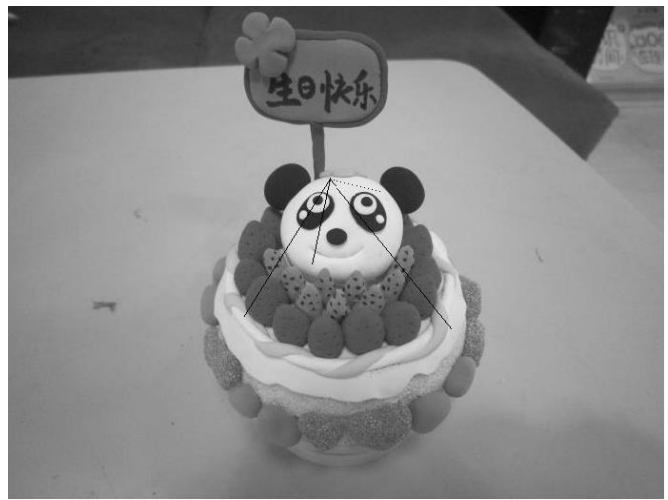

Figure 13. Matching Pixels in an Image

\subsection{Comparison for Copy-Move Authentication Approaches}

We also implemented the approaches with Ryu's [14] approach and Singh's [20] approach. We compared detection rate in duplicated rotation region of these approaches in Figure 14. 


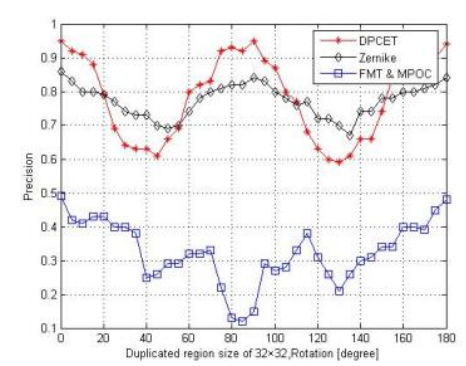

(a)

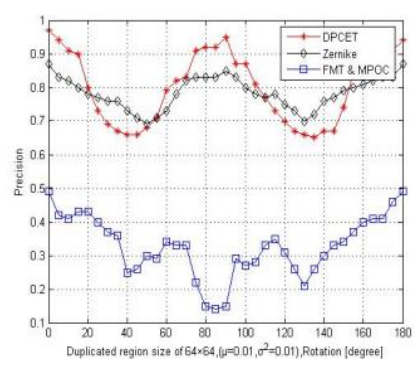

(b)

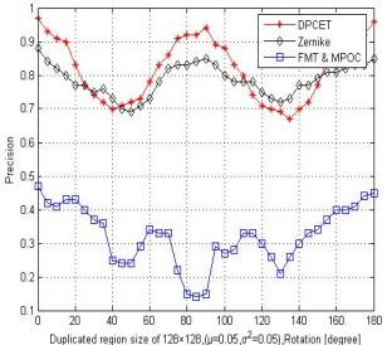

(c)

Figure 14. Detection precision rate of our DPCET approach and Zernike approach in [14], FMT \& MPOC approach in [20] with additive Gaussian noise from $0^{\circ} \sim 180^{\circ}$ rotated (separated by $5^{\circ}$ ). (a) Duplicated region size of $32 \times 32$ pixels; (b) Duplicated region size of $64 \times 64$ pixels; (c) Duplicated region size of $128 \times 128$ pixels.

Figure 14 shows our proposed approach is higher precision than FMT \& MPOC approach in [20], and in most case, average precision rate of our approach is also higher than Zernike approach in [14].

\section{Conclusions}

With the rapid development of computer technology, normal users can perform duplicated region operation easier for creating a realistic composite image. As such it is also significant to detect these duplicated operations for verifying and authenticating the originality of the digital images. In this paper, we have presented the overview of the advance techniques in image forensics and security applications. We have focused on the localization and detection of image duplicated forgery, for which we advocate Discrete Polar Harmonic Transforms as geometry feature representation of small duplicated overlapping image blocks which size is at least $32 \times 32$ pixels. For the geometric features, we proposed to construct rotation moment invariant and extract its rotation invariance. The detail formula derivation is discussed and analyzed in section 3. And the latter experiments can all be viewed in support of the above decision of formula derivation. All experimental results strongly suggest that localization and detection performance greatly depends on image geometric features, such as translation, rotation. The experiments in section 4.1 shows that the proposed approach can detect duplicated forgery images successfully, and furthermore, even if the duplicated forged image is subjected to rotation transform and additive Gaussian noise. When the size of duplicated region is larger than $32 \times 32$, we will achieve higher level of accuracy. The experiments in section 4.2 , we introduce the image False Detecting Rate (FDR) to authenticate misjudgment of our proposed approach in detecting nature or no being tampered image. In section 4.3, we compared the robustness of our approach with the previously proposed approaches which also research on duplicated rotation region in paper [14, [20]. Our proposed approach based on rotation moment invariants show more excellent. But the precision of our proposed approach in detecting of $(2 n+1) \pi / 4$ is not relatively high. This is due when the duplicated region or sub-image which is rotated to $(2 n+1) \pi / 4$ closely will led to change the relatively position of neighboring pixels which are realize to fill a certain pixel values greatly. In addition, we slide one pixel each time to detect the whole image which will lead to low efficiency. So in the next step, we will accelerate correlated work and improved detection approach to enhance robustness, increase precision and improve efficiency, make the approach for the duplicated rotation region of image detection more efficient. 


\section{Acknowledgments}

Corresponding Author: YANFEN GAN, Department of Information Science and Technology Guangdong University of Foreign Studies South China Business College, Guangzhou, 510540 P.R China, Emails: fannygyf@foxmail.com.

\section{References}

[1] X. Y. Pan and S. W. Lyu, Region Duplication Detection Using Image Feature Matching, IEEE Transaction on Information Forensics and Security, vol. 5, no. 4, (2010), pp. 857-867.

[2] J. Fridrich, D. Soukal and J. Lukás, “Authentication of Copy-Move Forgery In Digital Images," Proc. the Digital Forensic Research Workshop, (2003), pp. 55- 61.

[3] A. C. Popescu and H. Farid, "Exposing digital forgeries by detecting traces of resampling", IEEE Trans. Signal Processing, vol. 53, no. 2, (2005), pp. 758-767.

[4] Y. Cao, T. Gao, L. Fan and Q. Yang, "A robust detection algorithm for copy move forgery in digital images", Forensic Science international, vol. 214, (2012), pp. 33-43.

[5] Y. Huang, W. Lu, W. Sun, and D. Long, "Improved DCT-based detection of copy-move forgery in images," Forensic Science international, vol. 206, no.1, (2011), pp. 178-184.

[6] J. Zhang, Z.L. Feng and Y. T. Su, "New Approach for Detecting Copy-Move Forgery in Digital Images", Proc. Communication Systems (ICCS) 2008, China, (2008) November 19-21, pp. 362-366.

[7] S. Chakraborty, "Copy Move Image Forgery Detection Using Mutual Information", Proc. The Computing, Communications and Networking Technologies 2013, Tiruchengode, (2013) July 4-6, pp. 1-4.

[8] A. and S. D. Joshi, "Detection of Copy-Move Forgery Using Wavelet Decomposition", Proc. Signal Processing and Communication (ICSC) 2013, India, (2013) December 12-14, pp. 396-400.

[9] M. K. Hu, "Visual pattern recognition by moment invariants," IEEE Transaction on Information Theory, vol. 8, no. 2, (1962), pp. 179-187.

[10] C. Teh and R. Chin, "On Image Analysis by the Method of Moments", IEEE Transaction on Pattern Analysis and Machine Intelligence, vol. 10, no. 4, (1988), pp. 496-513.

[11] I. Amerini, L. Ballan, R. Caldelli and A. D. Bimbo, "A SIFT-Based Forensic Method for Copy-Move Attack Detection and Transformation Recovery", IEEE Transaction on Information Forensics and Security, vol. 6, no. 9, (2011), pp. 1099-1110.

[12] A. Sedaghat, M. Mokhtarzade and H. Ebadi, "Uniform Robust Scale-Invariant Feature Matching for Optical Remote Sensing Images", IEEE Transaction on Geoscience and Remote Sensing, vol. 49, no. 11, (2011), pp. 4516-4527.

[13] T. Chihaoui, S, Bourouis and K. Hamrouni, "Copy-move image forgery detection based on SIFT descriptors and SVD-matching”, Proc. Advanced Technologies for Signal and Image Processing (ATSIP) 2014, Tunisia, (2014) March 17-19, pp. 125-129.

[14] S. J. Ryu, M. J. Lee and H. K. Lee, "Detection of Copy-Rotate-Move Forgery Using Zernike Moments", Proc. International Workshop on Information Hiding (Lecture Notes in Computer Sciences) 2010, Germany, (2010), June 28-30, pp. 51-65.

[15] J. Revaud, G.Lavoue, and A.Baskurt, "Improving Zernike Moments Comparison for Optimal Similarity and Rotation Angle Retrieval”, IEEE Transactions on Pattern Analysis and Machine Intelligence, vol. 31, no. 4, (2009), pp. 627-636.

[16] B. J. Chen, H. Z. Shu, H. Zhang, G. Coatrieux,L. Luo, J. L. Coatrieux, "Combined Invariants to Similarity Transformation and to Blur Using Orthogonal Zernike Moments", IEEE Transaction on Image Processing, vol. 20 , no. 2 , (2011), pp. 345-360.

[17] S. Li, M. C. Lee, C. M. Pun, "Complex Zernike Moments Features for Shape-Based Image Retrieval", IEEE Transaction on Systems, Man, and Cybernetics-PART A: Systems and Humans, vol. 39, no. 1, (2009), pp. 227-237.

[18] S. Bayram, H. T. Sencar, and N. Memon, "An Efficient and Robust Method for Detecting Copy-move Forgery," Proc. IEEE International Conference on Acoustics, Speech and Signal Processing 2009, Taipei, Taiwan, (2009), April 19-24, pp. 1053-1056.

[19] Y. Sheng and L. Shen, "Orthogonal Fourier-Mellin moments for invariant pattern recognition", journal of the optical society of america a-optics image science and vision, vol. 11, no. 6, (1994), pp. 1748-1757.

[20] S. Singh, M. Ramalho, P. L. Correia and L. D. Soares, "Improved rotation-invariant degraded partial palmprint recognition technique", Proc. Biometrics and Forensics (IWBF) 2013, Portugal, (2013), April 4-5, pp. 1-4.

[21] P. T. Yap, X. D. Jiang and C. H. Kot, "Two-Dimensional Polar Harmonic Transforms for Invariant Image Representation," IEEE Transaction on Pattern Analysis and Machine Intelligence, vol. 32, no.7, (2010), pp. 1260-1270.

[22] L. D. Li, S. S. Li and J. Wang, "Copy-Move Forgery Detection Based on PHT", Proc. Information and Communication Technologies (WICT) 2012, India, (2012), October 30-November 2, pp. 1061-1065.

[23] Z. Yang and S. I. Kamata, "Fast Polar Harmonic Transforms", Proc. Control Automation Robotics \& Vision (ICARCV) 2010, Singapore, (2010), December 7-10, pp. 673-677. 
International Journal of Signal Processing, Image Processing and Pattern Recognition Vol.8, No.7 (2015) 